\title{
MINIMAL IDEALS IN GROUP RINGS
}

\author{
D. S. PASSMAN
}

AbSTRACT. Let $K[G]$ denote the group ring of $G$ over an algebraically closed field $K$. In this paper we show that $K[G]$ has a minimal left ideal which affords a finite dimensional representation of the ring if and only if $G$ is finite.

1. Annihilator ideals. Let $R$ be a ring with one and let $T$ be a subset of $R$. Then the left annihilator of $T, l_{R}(T)$, is defined by $l_{R}(T)=$ $\{\alpha \in R \mid \alpha T=0\}$. Clearly $l_{R}(T)$ is a left ideal of $R$. If in addition $T$ is a left ideal of $R$, then $l_{R}(T)$ becomes an ideal (two-sided).

Let $A$ be an ideal of $R$. We say that $A$ is an annihilator ideal if $A \neq R$ and if $A=l_{R}(T)$ for some subset $T$ of $R$. Observe that the condition $A \neq R$ is equivalent to $T \nsubseteq\{0\}$.

Let $K[G]$ denote the group ring of the group $G$ over the field $K$. We do not assume unless otherwise stated that $K$ is algebraically closed. If $A$ is an annihilator ideal in $K[G]$, then we let $\varphi_{A}$ denote the algebra homomorphism $\varphi_{A}: K[G] \rightarrow K[G] / A$. In this paper we study the $K$-algebra $K[G] / A$.

If $\alpha=\sum a_{x} x \in K[G]$, then we let Supp $\alpha$ be the finite subset of $G$ given by $\operatorname{Supp} \alpha=\left\{x \in G \mid a_{x} \neq 0\right\}$.

Lemma 1.1. Let $A$ be an annihilator ideal in $K[G]$ and set $G_{A}=$ $\left\{x \in G \mid \varphi_{A}(x) \in K\right\}$. Then $G_{A}$ is a finite normal subgroup of $G$.

Proof. Since $K$ is central in $K[G] / A$ it is clear that $G_{A}$ is a normal subgroup of $G$. Let $A=l_{K[G]}(T)$ and let $\alpha \in T, \alpha \neq 0$. If $x \in G_{A}$ then there exists $k \in K, \quad k \neq 0$, with $\varphi_{A}(x)=k=\varphi_{A}(k)$. Thus $x-k \in A$ so $(x-k) \alpha=0$. This yields $x \alpha=k \alpha$ so $x(\operatorname{Supp} \alpha)=\operatorname{Supp} \alpha$. We have therefore shown that $G_{A}$ permutes the finite nonempty set $\operatorname{Supp} \alpha \subseteq G$ by left multiplication and hence $G_{A}$ is finite.

The F.C. subgroup of $G$ is defined by

$$
\Delta=\Delta(G)=\left\{x \in G \mid\left[G: \mathbf{C}_{G}(x)\right]<\infty\right\} .
$$

Received by the editors January 29, 1971.

AMS 1970 subject classifications. Primary 16A26.

Key words and phrases. Group ring, minimal ideal, finite dimensional representation. 
We let $\theta: K[G] \rightarrow K[\Delta]$ denote the natural projection. The following is a simpler version of some work of M. Smith in [2].

TheOREM 1.2. Let $A$ be an annihilator ideal in $K[G]$. Let $\alpha \in K[G]$ and suppose that $\varphi_{A}(\alpha)$ is central in $K[G] / A$. Then $\alpha-\theta(\alpha) \in A$.

Proof. Say $A=l_{K[G]}(T)$ and let $\tau \in T$. Let $x \in G$. Then $\varphi_{A}(\alpha)$ commutes with $\varphi_{A}(x)$ so $\varphi_{A}(x \alpha-\alpha x)=0$ and $x \alpha-\alpha x \in A$. Thus

$$
1 x(\alpha \tau)-\alpha x \tau=(x \alpha-\alpha x) \tau=0 .
$$

Since this is true for all $x \in G$, Lemma 1.3 of [1] yields

$$
(\alpha-\theta(\alpha)) \tau=\theta(1) \alpha \tau-\theta(\alpha) \tau=0 .
$$

Since this is true for all $\tau \in T$ we have $\alpha-\theta(\alpha) \in l_{K[G]}(T)=A$ and the result follows.

Let $R$ be a ring with center $Z$. Then elements $\alpha_{1}, \alpha_{2}, \cdots, \alpha_{n} \in R$ are said to be linearly independent over $Z$ if $\eta_{1} \alpha_{1}+\eta_{2} \alpha_{2}+\cdots+\eta_{n} \alpha_{n}=0$ with $\eta_{i} \in Z$ implies that $\eta_{1}=\eta_{2}=\cdots=\eta_{n}=0$.

Corollary 1.3. Let $A$ be an annihilator ideal in $K[G]$. Then

(i) $\varphi_{A}(K[\Delta(G)])$ contains the center of $K[G] / A$.

(ii) $\varphi_{A}\left(x_{1}\right), \varphi_{A}\left(x_{2}\right), \cdots, \varphi_{A}\left(x_{n}\right)$ are linearly independent over the center of $K[G] / A$ if $x_{1}, x_{2}, \cdots, x_{n} \in G$ are in distinct cosets of $\Delta(G)$.

Proof. (i) Suppose $\varphi_{A}(\alpha)$ is central in $K[G] / A$. Then by Theorem 1.2, $\alpha-\theta(\alpha) \in A$. Thus $\varphi_{A}(\alpha)=\varphi_{A}(\theta(\alpha))$.

(ii) Suppose $\bar{\eta}_{1} \varphi_{A}\left(x_{1}\right)+\bar{\eta}_{2} \varphi_{A}\left(x_{2}\right)+\cdots+\bar{\eta}_{n} \varphi_{A}\left(x_{n}\right)=0$ with $\bar{\eta}_{i}$ in the center of $K[G] / A$. By part (i) there exists $\eta_{i} \in K[\Delta(G)]$ with $\bar{\eta}_{i}=\varphi_{A}\left(\eta_{i}\right)$. Thus the above becomes

$$
\varphi_{A}\left(\eta_{1} x_{1}+\eta_{2} x_{2}+\cdots+\eta_{n} x_{n}\right)=0 .
$$

Fix a subscript $j$ and set

$$
\begin{aligned}
\alpha & =\left(\eta_{1} x_{1}+\eta_{2} x_{2}+\cdots+\eta_{n} x_{n}\right) x_{j}^{-1} \\
& =\eta_{1} x_{1} x_{j}^{-1}+\eta_{2} x_{2} x_{j}^{-1}+\cdots+\eta_{n} x_{n} x_{j}^{-1} .
\end{aligned}
$$

Then $\varphi_{A}(\alpha)=0$ so $\varphi_{A}(\alpha)$ is central. Thus by Theorem 1.2 and the fact that the $x_{i}$ 's are in distinct cosets of $\Delta(G)$ we have $\alpha-\eta_{j}=\alpha-\theta(\alpha) \in A$. This yields $\bar{\eta}_{j}=\varphi_{A}\left(\eta_{j}\right)=\varphi_{A}(\alpha)=0$ and the result follows.

LEMMA 1.4. Let $A$ be an annihilator ideal in $K[G]$ and let $H$ be a subgroup of $G$. Then $B=A \cap K[H]$ is an annihilator ideal of $K[H]$ and therefore $\varphi_{A}(K[H])=\varphi_{B}(K[H])$.

Proof. Clearly $B=A \cap K[H]$ is an ideal in $K[H]$. Let $\left\{x_{v}\right\}$ be a set of right coset representatives for $H$ in $G$. Then every element $\alpha \in K[G]$ 
can be written uniquely as a finite sum $\alpha=\sum_{\nu} \alpha_{\nu} x_{v}$ with $\alpha_{v} \in K[H]$. If $\beta \in K[H]$ then clearly $\beta \alpha=0$ if and only if $\beta \alpha_{\nu}=0$ for all $\nu$. Thus if $A=l_{K[G]}(T)$ then it follows easily that $B=l_{K[H]}(S)$ where $S=$ $\left\{\tau_{v} \mid \tau \in T\right\} \subseteq K[H]$. This completes the proof.

2. Finite dimensional algebras. In this section we consider the possibility that $K[G] / A$ is a finite dimensional algebra over a field $F \supseteq K$.

Lemma 2.1. Let $G=\Delta(G)$ and let $A$ be an annihilator ideal in $K[G]$. Suppose that $K[G] / A$ is a finite dimensional algebra over a field $F \supseteq K$. Then there exists a subgroup $H$ of $G$ with $[G: H]<\infty$ and $\varphi_{A}(K[H])$ central in $K[G] / A$.

Proof. Set $E=K[G] / A$ and for each $x \in G$ let $V_{x}=\mathbf{C}_{E}\left(\varphi_{A}(x)\right)$. Then $V_{x}$ is certainly an $F$-subspace of $E$. Since $E$ is finite dimensional, it follows easily that there exists $x_{1}, x_{2}, \cdots, x_{n} \in G$ with

$$
\bigcap_{x \in G} V_{x}=V_{x_{1}} \cap V_{x_{2}} \cap \cdots \cap V_{x_{n}} \text {. }
$$

Set $H=\mathbf{C}_{G}\left(x_{1}\right) \cap \mathbf{C}_{G}\left(x_{2}\right) \cap \cdots \cap \mathbf{C}_{G}\left(x_{n}\right)$. Since $G=\Delta(G)$ we have $[G: H]<\infty$.

Let $y \in H$. Then $y$ centralizes $x_{1}, x_{2}, \cdots, x_{n}$ so $\varphi_{A}(y)$ centralizes $\varphi_{A}\left(x_{1}\right), \varphi_{A}\left(x_{2}\right), \cdots, \varphi_{A}\left(x_{n}\right)$ and hence

$$
\varphi_{A}(y) \in V_{x_{1}} \cap V_{x_{2}} \cap \cdots \cap V_{x_{n}}=\bigcap_{x \in G} V_{x} .
$$

Thus for all $x \in G, \varphi_{A}(y)$ centralizes $\varphi_{A}(x)$ and the result follows.

LEMMA 2.2. Let $A$ be annihilator ideal in $K[G]$ and suppose that $K[G] / A$ is a finite dimensional algebra over a field $F \supseteq K$. Then there exists an annihilator ideal $B \supseteq A$ in $K[G]$ such that $K[G] / B$ is a finite dimensional simple algebra over $F$.

Proof. By induction on $\operatorname{dim}_{F} K[G] / A$. Certainly if this dimension is 1 then the result follows with $B=A$. Suppose first that $K[G] / A$ is prime. Then since it is a finite dimensional algebra, it follows from the Wedderburn theorems that $K[G] / A$ is simple and we need only take $B=A$.

Suppose now that $E=K[G] / A$ is not prime. Then there exists $\bar{\alpha}$, $\bar{\beta} \in E-\{0\}$ with $\bar{\alpha} E \bar{\beta}=0$. Let $\bar{\alpha}=\varphi_{A}(\alpha), \bar{\beta}=\varphi_{A}(\beta)$ and say $A=$ $l_{K[G]}(T)$. Since $\bar{\beta} \neq 0$ we have $\beta T \neq 0$ and thus $S=K[G] \beta T \neq 0$. Then $S$ is a left ideal in $K[G]$ so $C=l_{K[G]}(S)$ is an annihilator ideal in $K[G]$. Now $A$ is an ideal so

$$
A S=(A K[G] \beta) T \subseteq A T=0
$$

and hence $C \supseteq A$. Moreover, $\varphi_{A}(\alpha K[G] \beta)=0$ so

$$
\alpha S=(\alpha K[G] \beta) T \subseteq A T=0
$$


and $\alpha \in C$. Thus $C>A$ and $K[G] / C$ is a proper homomorphic image of $E$. This implies that $K[G] / C$ is an $F$-algebra and $\operatorname{dim}_{F} K[G] / C<$ $\operatorname{dim}_{F} K[G] / A$. By induction there exists an annihilator ideal $B \supseteq C$ with $K[G] / B$ simple. Since $B \supseteq C \supseteq A$, the result follows.

LEMMA 2.3. Let $x \in G$ have infinite order. Then $\bigcap_{n=1}^{\infty}\left(x^{n}-1\right) K[G]=0$.

Proof. Let $\left\{y_{v}\right\}$ be a set of right coset representatives for $\langle x\rangle$ in $G$. Then every element of $K[G]$ can be written uniquely as a finite sum $\alpha=\sum_{v} \alpha_{v} y_{v}$ with $\alpha_{v} \in K[\langle x\rangle]$. Thus $\alpha \in \bigcap_{n=1}^{\infty}\left(x^{n}-1\right) K[G]$ if and only if $\alpha_{v} \in \bigcap_{n=1}^{\infty}\left(x^{n}-1\right) K[\langle x\rangle]$ for all $\nu$. Therefore it suffices to show that the latter intersection is zero or in other words we can assume that $G=\langle x\rangle$.

Let $\alpha \in K[G], \alpha \neq 0$. Then Supp $\alpha$ is a finite subset of $\langle x\rangle$ and we let

$$
\max (\alpha)=\max \left\{a \mid x^{a} \in \operatorname{Supp} \alpha\right\}, \quad \min (\alpha)=\min \left\{a \mid x^{a} \in \operatorname{Supp} \alpha\right\} .
$$

Now clearly if $\alpha \in\left(x^{n}-1\right) K[G], \alpha \neq 0$ then $\max (\alpha)-\min (\alpha) \geqq n$ and this therefore yields $\bigcap_{n=1}^{\infty}\left(x^{n}-1\right) K[G]=0$.

LEMMA 2.4. Let $A$ be an annihilator ideal in $K[G]$ and suppose that $K[G] / A$ is a field. Then $G$ is a periodic group.

Proof. Suppose by way of contradiction that $G$ has an element $x$ of infinite order and let $A=l_{K(G)}(T)$. Then for each integer $n \geqq 1, x^{n}-1$ is not a zero divisor in $K[G]$ by Lemma 2.4 of [1] so $x^{n}-1 \notin A$ and $\varphi_{A}\left(x^{n}-1\right) \neq 0$. Since $K[G] / A$ is a field there exists $\beta_{n} \in K[G]$ with $\varphi_{A}\left(x^{n}-1\right) \varphi_{A}\left(\beta_{n}\right)=1$. Thus $\varphi_{A}\left(\left(x^{n}-1\right) \beta_{n}-1\right)=0$. If $\tau \in T$ then this yields

$$
\left(x^{n}-1\right) \beta_{n} \tau-\tau=\left[\left(x^{n}-1\right) \beta_{n}-1\right] \tau=0
$$

so $\tau \in\left(x^{n}-1\right) K[G]$. Since this is true for all $n \geqq 1$ and all $\tau \in T$ we have $T \subseteq \bigcap_{n=1}^{\infty}\left(x^{n}-1\right) K[G]=0$, by Lemma 2.3, a contradiction.

3. Finite dimensional representations. We now come to the main result of this paper.

THEOREM 3.1. Let $K[G]$ denote the group ring of $G$ over an algebraically closed field $K$ and let $A$ be an annihilator ideal in $K[G]$. If $K[G] / A$ is a finite dimensional algebra over a field $F \supseteq K$, then $G$ is finite.

Proof. We first reduce the problem to $\Delta(G)$. By Corollary 1.3 (ii) we have $[G: \Delta]<\infty$. Also by Corollary $1.3(\mathrm{i}), \varphi_{A}(K[\Delta])$ is a subring of $\varphi_{A}(K[G])$ containing the center. Thus $\varphi_{A}(K[\Delta]) \supseteq F$ so $\varphi_{A}(K[\Delta])$ is also a finite dimensional $F$-algebra. Finally by Lemma 1.4 there exists an annihilator ideal $B$ in $K[\Delta]$ with $\varphi_{A}(K[\Delta])=\varphi_{B}(K[\Delta])$. Therefore $K[\Delta]$ satisfies all the hypotheses of this theorem and since $[G: \Delta(G)]<\infty$ it suffices to consider the case $G=\Delta(G)$. 
We now assume that $G=\Delta(G)$ and by Lemma 2.2 we can assume that $A$ is so chosen that $K[G] / A$ is simple. Then $K[G] / A$ is a finite dimensional simple algebra so its center $Z$ is a field. Now by Lemma $2.1, G$ has a subgroup $H$ with $[G: H]=n<\infty$ and with $\varphi_{A}(K[H])$ central in $K[G] / A$. Thus $\varphi_{A}(K[H])$ is an integral domain but it is in fact a field. Let $x_{1}, x_{2}, \cdots$, $x_{n}$ be a set of right coset representatives for $H$ in $G$. Then $K[G]=$ $\sum_{1}^{n} K[H] x_{i}$ so $K[G] / A$ is a finitely generated module over $\varphi_{A}(K[H])$. Therefore every element of $K[G] / A$ is integral over $\varphi_{A}(K[H])$. In particular $Z$ is a field which is integral over $\varphi_{A}(K[H]) \subseteq Z$ and this implies (by looking at the integral equation satisfied by the reciprocals of the elements of $\left.\varphi_{A}(K[H])-\{0\}\right)$ that $\varphi_{A}(K[H])$ is also a field. By Lemma 1.4 there exists an annihilator ideal $B$ in $K[H]$ with $\varphi_{A}(K[H])=\varphi_{B}(K[H])$. Since $[G: H]<\infty$ it suffices to show that $H$ is finite.

Thus we have reduced the problem to the case in which $K[G] / A$ is in fact a field. By Lemma 2.4, $G$ is periodic. Now let $x \in G$. Then $x^{n}=1$ for some $n>1$ so $\varphi_{A}(x)$ is algebraic over $K$. Since $K$ is algebraically closed this implies that $\varphi_{A}(x) \in K$. Thus in the notation of Lemma $1.1, G=G_{A}$ and by that lemma $G$ is finite. This completes the proof of the theorem.

Let $E$ be an algebra over a field $K$. An irreducible representation $\rho$ of $E$ is said to be finite dimensional if $\rho(E)$ satisfies a polynomial identity over $K$.

COROLlaRY 3.2. Let $K[G]$ be the group ring of $G$ over an algebraically closed field $K$. Then $K[G]$ has a minimal left ideal which affords a finite dimensional representation of the ring if and only if $G$ is finite.

Proof. Suppose first that $G$ is finite. Then $K[G]$ is a finite dimensional algebra so it has minimal left ideals. Let $I$ be such an ideal. Then $I$ affords a finite dimensional representation of $K[G]$ since $K[G]$ satisfies a polynomial identity.

Conversely suppose that $K[G]$ has a minimal left ideal $I$ which affords a finite dimensional representation. Let $A$ denote the kernel of the homomorphism $\rho: K[G] \rightarrow$ End $(I)$. Then $A$ is clearly the set of left annihilators of $I$ so $A$ is an annihilator ideal and $\rho=\varphi_{A}$. Now $\varphi_{A}(K[G])$ is a primitive ring satisfying a polynomial identity and hence by a theorem of Kaplansky (Theorem 6.4 of $[1]) \varphi_{A}(K[G])$ is a finite dimensional algebra over some field $F \supseteq K$. By Theorem 3.1, $G$ is finite.

Finally we show by example that the above is false if $K$ is not algebraically closed. Let $K=Q$ be the field of rationals and let $G$ be the $P_{\infty}$ group for some prime $p$. Thus

$$
\left.G=\left\langle x_{1}, x_{2}, x_{3}, \cdots, x_{n}, \cdots\right| x_{1}^{p}=1, x_{n+1}^{p}=x_{n} \text { for all } n \geqq 1\right\rangle .
$$

Define $p^{n}$ th roots of unity in the complex numbers $C$ inductively by $\mathcal{E}_{1}$ is a primitive $p$ th root of 1 and $\varepsilon_{n+1}^{p}=\mathcal{E}_{n}$. Then the map $\varphi: Q[G] \rightarrow C$ 
given by $x_{i} \rightarrow \varepsilon_{i}$ is clearly a homomorphism of $Q[G]$ onto a subfield $F$ of $C$. We compute the kernel of this map.

Set

$$
e=(1 / p)\left(1+x_{1}+x_{1}^{2}+\cdots+x_{1}^{p-1}\right) .
$$

Then $e$ is an idempotent in $Q[G]$ and $\varphi(e)=0$ since $1+\varepsilon_{1}+\varepsilon_{1}^{2}+\cdots+$ $\varepsilon_{1}^{p-1}=0$. Thus the kernel of $\varphi$ contains $e Q[G]$. Now let $\varphi(\alpha)=0$. Then there exists an integer $n \geqq 1$ with $\alpha \in Q\left[\left\langle x_{n}\right\rangle\right]$ and we can write $\alpha=\sum_{i \geqq 0} a_{i} x_{n}^{i}$. Thus $0=\varphi(\alpha)=\sum_{i \geqq 0} a_{i} i_{n}^{i}$ and it follows that $\sum a_{i} x_{n}^{i}$, viewed as a polynomial in $x_{n}$, is divisible by the cyclotomic polynomial

$$
1+x_{n}^{p^{n-1}}+x_{n}^{2 p^{n-1}}+\cdots+x_{n}^{(p-1) p^{n-1}}=p e .
$$

Hence $\alpha \in e Q[G]$ and $e Q[G]$ is the kernel of $\varphi$.

Since $Q[G]=(1-e) Q[G]+e Q[G]$ we see that $(1-e) Q[G] \simeq F$. Thus $I=(1-e) Q[G]$ is a minimal ideal in $Q[G]$ which affords a finite dimensional (in fact, commutative) representation of $Q[G]$. Since $G$ is not finite, this yields the required counterexample.

\section{REFERENCES}

1. D. S. Passman, Infinite group rings, Marcel Dekker, New York, 1971.

2. M. Smith, On group algebras, Bull. Amer. Math. Soc. 76 (1970), 780-782.

Department of Mathematics, University of Wisconsin, Madison, Wisconsin 53706 